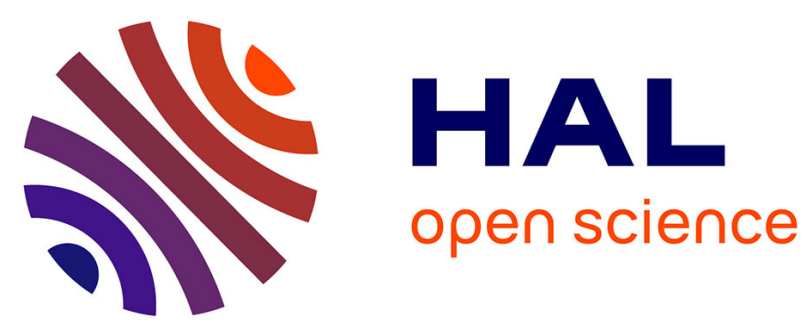

\title{
Une inscription sur bronze trouvée dans l'établissement gallo-romain de L'Auribelle-Basse à Pézenas (Hérault)
}

Michel Christol, Stéphane Claude Mauné

\section{To cite this version:}

Michel Christol, Stéphane Claude Mauné. Une inscription sur bronze trouvée dans l'établissement gallo-romain de L'Auribelle-Basse à Pézenas (Hérault). Gallia - Archéologie de la France antique, 2003, 60, pp.369-382. 10.3406/galia.2003.3058 . halshs-00507436

\section{HAL Id: halshs-00507436 \\ https://shs.hal.science/halshs-00507436}

Submitted on 9 Jan 2020

HAL is a multi-disciplinary open access archive for the deposit and dissemination of scientific research documents, whether they are published or not. The documents may come from teaching and research institutions in France or abroad, or from public or private research centers.
L'archive ouverte pluridisciplinaire HAL, est destinée au dépôt et à la diffusion de documents scientifiques de niveau recherche, publiés ou non, émanant des établissements d'enseignement et de recherche français ou étrangers, des laboratoires publics ou privés.

\section{(1) (1) $\$$}

Distributed under a Creative Commons Attribution - NonCommercial - NoDerivatives $\mid 4.0$ 


\title{
UNE INSCRIPTION SUR BRONZE TROUVÉE DANS L'ÉTABLISSEMENT GALLO-ROMAIN DE L'Auribelle-Basse à PÉzenas (HÉrAuLT)
}

\author{
Michel Christol ${ }^{*}$ et Stéphane MaunÉ**
}

\begin{abstract}
Mots-clés. Gaule Narbonnaise, période tardo-républicaine et augustéenne, inscription latine, plaque en bronze, contrat d'hospitalité.
Résumé. L'établissement de L'A uribelle-Basse à Pézenas (Hérault), en Gaule Narbonnaise, a livré plusieurs fragments d'une plaque en bronze comportant un texte latin daté de la période tardo-républicaine ou augustéenne, ce qui consititue une découverte d'un grand intérêt, surtout lorsque l'on connaît la grande rareté de ce type de support pour l'épigraphie provinciale. Avant d'analyser son contenu, les auteurs s'attachent à souligner les spécificités de ce site et se proposent de réfléchir sur l'interprétation que l'on peut en faire. Installé dans le courant de la seconde moitié du Irr s. avant J.-C., période à laquelle est rattachée l'inscription, cet établissement reste occupé jusque vers le milieu du III's. C'est de l'un de ses niveaux d'abandon que provient la majorité des fragments de la plaque.

L'analyse du texte montre qu'il peut s'agir d'un contrat d hospitium comportant une série de noms celtiques offrant des parallèles en Narbonnaise mais aussi ailleurs en Gaule. In fine, les auteurs s'interrogent sur le contexte de la découverte : contemporaine de l'édification de l'établissement, cette plaque a en effet été volontairement brisée avant que certains morceaux aient été jetés dans un dépotoir et d'autres dispersés autour du secteur bâti, près de 300 ans après.
\end{abstract}

Key-words. Gallia Narbonensis, Late-Republican and Augustan period, Latin inscription, bronze tablet, hospitium.

Abstract. The discovery in the establishment of L'Auribelle-Basse at Pézenas, in Gallia Narbonensis, of several pieces of a bronze tablet bearing a Latin text dated from the Late-Republican and Augustan period, is of great interest, especially since this kind of material is scarcely found in provincial epigraphy. First, the authors draw attention to the specific features of the site and try to propose an interpretation. Built during the second half of the $I^{\text {st }} B C$, to which the inscription belongs, this establishment continues to be occupied until around the mid third century. The majority of the pieces has been recovered in one of the demolition layers. The analysis of the content shows that the text could be a hospitium contract recording a series of Celtic names with parallels in Narbonensis but also in other parts of Gaul. Finally, the context is being examined: contemporary to the setting of the establishment, this tablet has deliberately been broken before some of the pieces being thrown in a rubbish-pit and others scattered all around the building near 300 years later.

Schlagwörter Gallia Narbonensis, spätrepublikanisch-augusteische Zeit, lateinische Inschrift, Bronzetäfelchen, Gastfreundschaftsvertrag.

Zusammenfassung. Auf dem Gelände der römischen Siedlung von L'Auribelle-Basse bei Pézenas (Dép. Hérault) kamen mehrere Fragmente eines Bronzetäfelchens mit einem lateinischen Text aus spätrepublikanisch-augusteischer Zeit ans Licht. Der Fund darf angesichts der bekannten Seltenheit derartiger Inschriftenträger innerhalb der epigraphischen Überlieferung der römischen Provinzen als bedeutsame Entdeckung gelten.

\footnotetext{
* Universitć Paris 1/Panthéon-Sorbonne et UMR 8585 du CNRS, Centre de recherches Gustave-Glotz, 17 rue de la Sorbonne, F-75231 Paris Cedex 05.

** UMR 154 du CNRS, 390 avenue de Pérols, F-34970 Lattes. Mél : stcphane.maune@cnrs-mop.fr
} 
Zunächst bemühen sich die Verf. darum, die Besonderheiten des Fundplatzes herauszuarbeiten und stellen grundsätzliche Überlegungen zu seiner Deutung an. Die Anlage wird im Verlauf der zweiten Hälfte des ersten Jahrhunderts v. Chr. errichtet (der Teil, der auch die Inschrift eigentlich zuzuweisen ist), und bleibt bis gegen die Mitte des dritten Jahrhunderts n. Chr. in Benutzung. Der Großteil der Inschriftenfragmente stammt aus einem Horizont der Spätphase, in der die Siedlung aufgegeben wird.

Wie eine kritische Analyse des Schriftstückes zeigt, handelt es sich möglicherweise um einen Gastfreundschaftsvertrag. Der Text weist eine Reihe keltischer Namen auf, zu denen es Parallelen in der Narbonensis, aber auch anderswo in Gallien gibt. Abschließend werden Überlegungen zum Zustandekommen des Befundes angestellt. Man hatte den Gegenstand aus der Bauzeit der Anlage absichllich zerbrochen. Knapp drei Jahrhunderte nach der Anfertigung der Tafel wurden ihre Bruchstücke achtlos fortgeworfen und teilweise im Siedlungsbereich verstreut.

Übersetzt vom Stefan WIRTH

Au printemps 1998, une prospection de surface, effectuée sur l'emplacement du site gallo-romain de L'Auribelle-Basse à Pézenas (Hérault) (fig. 1), a permis la découverte d'un petit fragment d'une plaque en bronze portant une inscription incomplète répartie sur quatre lignes (voir fragment $n^{\circ} 4$, fig. 7 p. 376). Quelques mois plus tard, un deuxième fragment de la même inscription, comportant cette fois-ci le départ de deux lignes, fut mis au jour à environ $10 \mathrm{~m}$ du précédent (voir fragment $\mathrm{n}^{\circ} 5$, fig. 8 p. 376). L'analyse préliminaire de ces premiers éléments - reposant sur la graphie ancienne - a montré qu'ils appartenaient à un texte écrit entre la fin de la période tardo-républicaine et le changement d'ère (Christol, Mauné, 2000).

Cette découverte et l'intérêt présenté par l'étude préliminaire de ce site ont suscité la mise en place d'un projet de fouille programmée extensive, pluriannuelle, engagé en $2000^{1}$. C'est alors que furent mis au jour, dans un vaste dépotoir du $\mathrm{III}^{\mathrm{c}} \mathrm{s}$. après J.-C., plusieurs autres fragments de la plaque qui ont confirmé la chronologie haute du texte, notamment grâce à l'emploi de la forme legatei. Rares sont les inscriptions sur bronze trouvées en Gaule, si bien qu'il a paru intéressant de présenter ces documents, même si des découvertes ultérieures peuvent corriger les hypothèses proposées. En préambule à cette analyse, il nous a semblé intéressant de résumer les connaissances et hypothèses actuelles sur l'établissement de L'Auribelle-Basse. Elles peuvent en effet éclairer la nature et la fonction de ce texte.

1. Les données présentécs ici s'inscrivent plus largement dans un programme collectif de recherche pluriannuel du ministère de la Culture/service régional de l'archéologie Languedoc-Roussillon: Formes et dynamiques de l'occupation du sol en Transalpine occidentale aux If et $I^{e r} s$. av. J.-C., rassemblant une dizaine d'historiens et archéologues de l'université de Besançon et des UMR 154 (Lattes) et 8585 (Paris) du CNRS. L'intérêt du site et la découverte, en surface, de fragments de la plaque en bronze qui fait l'objet du présent article ont entrainé l'achat, par la communauté de communes du Pays de Pézenas, des 1,2 ha de terrain entourant la villa. Désormais propriété d'une collectivité locale, le site, protégé, pourra être finement fouillé et étudié.

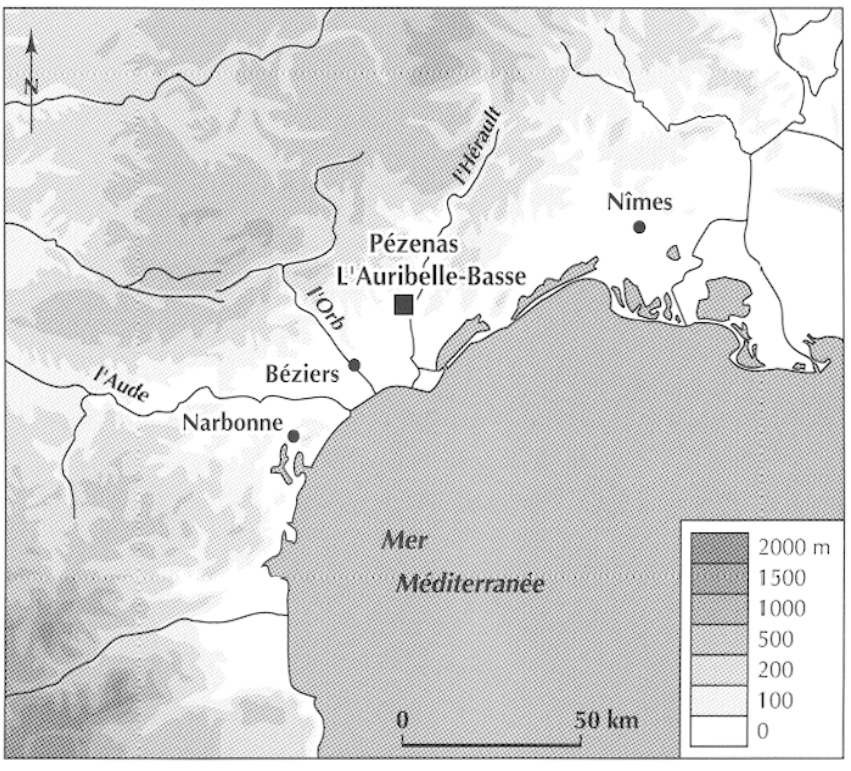

Fig. 1 - Localisation de l'établissement de L'Auribelle-Basse à Pézenas (Hérault), en Narbonnaise (fond de carte (C) M. Py, CNRS).

\section{LE LIEU DE DÉCOUVERTE : UN ÉTABLISSEMENT DE NARBONNAISE CENTRALE}

Le site de L'Auribelle-Basse est implanté à proximité d'une petite rivière, le Rieutort, sur un terrain en pente douce (alt. $35 \mathrm{~m}$ ), orienté au sud-est, et qui offre un large panorama sur la vallée de la Peyne, affluent de l'Hérault, qui coule à environ $500 \mathrm{~m}$ plus au sud. Dans cette microrégion du territoire de la cité de Béziers, les recherches archéologiques menées depuis une dizaine d'années éclairent la densité de l'occupation du sol et l'importance de l'exploitation des ressources locales entre le début du $\mathrm{I}^{\mathrm{er}} \mathrm{s}$. avant J.-C. et le courant du III ${ }^{\mathrm{e}} \mathrm{s}$. après J.-C. De nombreux sites sont en cffct connus dans un rayon de $1500 \mathrm{~m}$ (fig. 2), qu'il s'agisse de simples établissements ruraux ou de villae comme celle 
de La Perrière, qui aurait livré de la statuaire en marbre, ou celle de Saint-Jean-de-Bébian (Pézenas) près de laquelle a été découverte anciennement une plaque de colombarium portant les tria nomina de A. Poblicius Felix (Mauné, 1998, p. 420-429).

\section{LA CHRONOLOGIE D'OCCUPATION : UNE CRÉATION COLONIALE PRÉAUGUSTÉENNE OU AUGUSTÉENNE ?}

La datation de L'Auribelle-Basse (seconde moitié du $\mathrm{I}^{\text {er }}$ s. avant J.-C./milieu du III' s. après J.-C.) est fournie par l'analyse du mobilier collecté en surface (2 500 fragments de céramique) mais aussi par celui, plus abondant, livré par les deux campagnes de fouille (30000 fragments). À ceci s'ajoutent les monnaies et le petit mobilier qui couvrent toute la période considérée. Nous ne nous intéresserons ici qu'aux périodes de fondation et d'abandon du site car elles concernent très directement l'inscription sur bronze.

La présence d'un petit lot de céramique campanienne $\mathrm{B}$ et $\mathrm{C}$, de sigillée italique, d'amphores de Tarraconaise Pascual 1 et Dressel 2-4, d'Haltern 70 de Bétique, de Dressel $1 \mathrm{~b}$ italique ainsi que de céramique à vernis rouge pompéien fixe les débuts de l'occupation dans le courant de la seconde moitié du $\mathrm{I}^{\mathrm{er}} \mathrm{s}$. avant J.-C. L'Auribelle-Basse appartient à cette génération encore mal connue des établissements fondés durant une période capitale du point de vue de l'évolution institutionnelle et politique locale. Après l'attribution du droit latin à plusieurs cités indigènes locales, situées dans le bassin de l'Hérault - Cessero, Piscinae, Lutevala déduction coloniale de Béziers par Octave en 36 avant J.-C. a pu constituer un épisode historique majeur pour cette petite région. La création d'un corps civique mixte comprenant des vétérans de la VII légion et des indigènes ayant bénéficié du droit romain (Clavel, 1970) a pu, en effet, entrainer, avec l'installation des nouveaux colons, des bouleversements dans les structures de l'occupation du sol et de la possession de la terre, ce dont rendrait compte, notamment, la centuriation coloniale Béziers C, bien présente dans la vallée de la Peyne (Clavel-Lévêque, 1998).

La date d'abandon est fixée par le mobilier le plus récent collecté en surface mais surtout grâce à la fouille d'un vaste dépotoir (FS 1043) venu combler une grande fosse située à l'arrière des bâtiments d'habitation. L'abondant mobilier recueilli dans ce niveau sombre comprend plus de 7500 fragments de céramique, un lot très important de restes de faune ainsi que des petits objets, fragmentés pour la plupart. C'est au milieu de ces débris, mêlés à un sédiment sombre riche en cendre, que furent trouvés six des huit fragments de la plaque.
Comme plus des deux tiers des établissements ruraux de la région de Béziers, L'Auribelle-Basse n'est pas parvenu à surmonter les difficultés qui ont touché les campagnes de Gaule Narbonnaise entre le milieu du $\mathrm{II}^{\mathrm{c}}$ s. et la fin du III ${ }^{\mathrm{e}} \mathrm{s}$. après J.-C. (Fiches dir., 1996). Dans la vallée de l'Hérault, ce phénomène touche environ $65 \%$ des établissements occupés précédemment, et l'on n'enregistre aucune création entre la fin du I ${ }^{\mathrm{er}} \mathrm{s}$. et le courant du III ${ }^{\mathrm{c}} \mathrm{s}$.

\section{DES CARACTÉRISTIQUES ORIGINALES}

Le site de L'Auribelle-Basse a été découvert en octobre 1996 par les membres du club archéologique de MontagnacPézenas, lors d'une prospection de surface effectuée aux abords du domaine de Roquelune (Mauné, 1998, p. 490-492). Sur un peu plus de $4000 \mathrm{~m}^{2}$, le mobilier, ramassé au milieu de nombreux fragments de matériaux de construction, a permis d'identifier un habitat gallo-romain présentant, a priori, toutes les caractéristiques d'une villa ${ }^{2}$ disposant d'une série d'équipements résidentiels. L'abondance des fragments de marbre - environ 180 - de tailles et de fonctions diverses, la découverte de fragments de tubulures d'hypocauste, de tesselles éparses de mosaïque, de verre à vitre et d'enduit peint polychrome soulignent l'importance de l'investissement immobilier réalisé par le propriétaire. On peut également ajouter à ces éléments un aqueduc mis au jour en trois endroits différents, situés à environ $1500 \mathrm{~m}$ en amont du site, dont la direction et la pente indiquent qu'il s'agit bien d'un élément conduisant à cet établissement et lui appartenant. Compte tenu de son débit $(84 \mathrm{l} / \mathrm{s})$, on pouvait supposer que cet ouvrage était destiné, en plus de l'alimentation en eau courante, à l'approvisionnement d'un balnéaire.

L'Auribelle-Basse constitue à l'évidence un site particulier car il occupe une assez faible superficie, en contradiction avec une interprétation de type villa. En effet, les villae du Haut-Empire connues dans la région de Béziers et plus particulièrement dans la vallée de l'Hérault occupent toutes de vastes superficies, égales ou supérieures à 1 ha (Mauné, 1998, p. 97-102 ; Pellecuer, 2000, p. 361-366) : dans l'Hérault, les villae fouillées de La Domergue à Sauvian (Ginouvez, 1995), des Prés-Bas à Loupian (Pellecuer, 2000)

2. On ne s'attardera pas ici sur les questions relatives à l'emploi du terme villa, qui fait l'objet, pour la Gaule méridionale, d'un assez large consensus au sein de la communauté archéologique (Pellecuer dir., 1996). Il s'agit toujours de grands ou très grands établissements, couvrant la plupart du temps plus de 1 ha, et qui présentent des vestiges de pars urbana bien individualisés. Cependant, des exceptions, de plus en plus nombreuses, dont L'Auribelle-Basse, apparaissent au fur et à mesure des découvertes et font l'objet de débats et de commentaires. 
ou de Vareilles à Paulhan (Mauné, à paraître) illustrent assez bien cette tendance, perceptible ailleurs en Narbonnaise, par exemple dans l'actuel département du Var (Brun, 1999, p. 150 et index).

La grande rareté d'éléments de dolia constitue une autre spécificité du dossier et pose, cette fois-ci, le problème de la vocation du site; en effet, dans la vallée de la Peyne, les établissements du Haut-Empire livrent pratiquement tous des vestiges d'installations vinicoles.

Faut-il plaider ici en faveur d'autres activités que l'archéologie ne parvient pas à illustrer de manière satisfaisante ? Les activités productives principales étaient-elles localisées ailleurs que sur l'emprise de l'habitat? Sommesnous en présence d'une villa ou bien d'un établissement ayant connu un évolution de son statut et de sa fonction durant ses trois siècles d'occupation?

\section{UN DOMAINE COMPORTANT UN PÔLE RÉSIDENTIEL ET PLUSIEURS PÔLES PRODUCTIFS ?}

L'hypothèse la plus séduisante serait peut-être de considérer L'Auribelle-Basse comme le siège d'un domaine du Haut-Empire constitué de plusieurs entités. Ne rassemblant pas les bâtiments nécessaires à la production et au stockage agricoles, le siège du domaine, lieu de résidence permanent ou temporaire du propriétaire, n'occuperait alors qu'une superficie réduite.

Si l'on poursuit dans cette voie, les établissements de Roquelune (fig. 2, $\mathrm{n}^{\circ}$ 2) et du Traou-del-Counil-Ouest (fig. $2, \mathrm{n}^{\circ} 3$ ), situés respectivement à $450 \mathrm{~m}$ au sud et au nord, dans le même bassin-versant, pourraient alors être considérés comme les lieux de production et de stockage du domaine. Le premier est occupé entre le début du $\mathrm{I}^{\text {er }} \mathrm{s}$. avant J.-C. et le milieu du $\mathrm{II}^{\mathrm{e}}$ s. après J.-C. ; il a livré des éléments de nombreux dolia, des fragments de meules en basalte et plusieurs pesons de tisserand ${ }^{3}$ qui indiquent la double vocation du site, agricole et artisanale (Mauné, 1998, p. 423). Le second est, quant à lui, occupé entre la première moitié du $\mathrm{I}^{\mathrm{cr}} \mathrm{s}$. et la fin du $\mathrm{III}^{\mathrm{e}} \mathrm{s}$. après J.-C., et l'abondance des fragments de dolia suggère également d'y localiser un chai vinicole ; enfin, l'élément le plus intéressant est relatif à la proximité immédiate d'un front de carrière ayant servi à extraire du calcaire coquillier dont le grain et l'aspect sont très proches de ceux des éclats de moellons trouvés sur l'emprise de L'Auribelle-Basse (id., ibid., p. 426-427). Sur ces

3. Installé contre une petite rivière, le site a récemment livré un fragment d'amphore à alun de type Richıboruugh 527 qui confirme la présence d'installations liées au travail de la laine. deux sites couvrant chacun environ $1000 \mathrm{~m}^{2}$, la présence de fragments d'amphores, de céramiques fines et de monnaies ${ }^{4}$ nous renseigne enfin sur leur statut : il ne s'agit pas de simples annexes, mais bien d'établissements ruraux, peut-être satellites de la villa voisine dont la vocation serait alors essentiellement résidentielle. La constitution de cette entité, composée d'éléments mis en place à des époques différentes, se serait faite entre la période augustéenne et la seconde moitié du $\mathrm{I}^{\mathrm{er}}$ s. après J.-C., comme tend à le montrer la confrontation entre les deux cartes de la figure 2.

À partir de ces éléments, on pourrait prudemment rattacher au fundus ainsi défini les terres situées plus en amont, le long du cours supérieur du Rieutort, de part et d'autre de l'aqueduc. Celui-ci pouvait capter - mais il faudra s'en assurer - les eaux d'une importante source pérenne. située à $1200 \mathrm{~m}$ de la villa et près de laquelle on a repéré, en 1999, les vestiges d'une installation du Haut-Empire (fig. 2, $\mathrm{n}^{\circ}$ 5) faisant face à un quatrième établissement (fig. 2, $n^{\circ} 4$ ), celui de Saint-Martin (Mauné, 1998, p. 341). Se dessinerait ainsi une vaste zone, axée sur le bassin-versant du Rieutort et sur le tracé de l'aqueduc ${ }^{5}$, dont on peut supposer qu'elle aurait constitué le territoire de la villa de L'Auribelle-Basse durant le Haut-Empire. La densité et la grande proximité topographique de quatre établissements contemporains ${ }^{6}$, ainsi que l'apparente cohérence de l'occupation du sol laissent penser que l'on aurait affaire ici à un domaine d'une certaine ampleur, atteignant environ 300 ha. Celui-ci était peut-être exploité à partir de plusieurs pôles distincts ; il disposait d'un centre domanial, certes de petite taille mais présentant toutes les caractéristiques d'une demeure résidentielle. Dans ce schéma, la villa constituerait, au milieu du III $^{\mathrm{c}}$ s., le seul site encore occupé dans ce bassinversant ${ }^{7}$. Voilà résumées les hypothèses de travail ayant servi de cadre de réflexion juste avant que débute une fouille programmée pluriannuelle.

La première campagne de fouille avait pour objectif d'évaluer les potentialités précises du site ainsi que l'état de conservation des vestiges et leur extension ${ }^{8}$.

4. Roquelune a livré un quinaire d'Egnatuleius, daté de 97 avant J.-C., unc moitié d'as oncial du $\mathrm{II}^{\mathrm{e}} \mathrm{-}^{\mathrm{er}} \mathrm{s}$. avant J.-C., un quadrans de type indéterminé de la première moitié du $\mathrm{I}^{\mathrm{er}} \mathrm{s}$. avant J.-C., deux demi-as de Nîmes et un as de Vienne, un demi-dupondius au crocodile, un as du Haut-Empire indéterminé et un as de Claude ; Lou Traou-del-CounilOuest, une monnaie à tête radiée du $\mathrm{III}^{\mathrm{e}} \mathrm{s}$., indéterminée.

5. Le tracé de celui-ci constituant ainsi l'épine dorsale de la propriété.

6. L'analyse porte ici sur la période qui s'étend entre le milieu du $I^{\mathrm{cr}} \mathrm{s}$. et du II $s$. après J.-C.

7. Quelques traces du III" s. sont cependant attestées sur le site voisin du Traou-dcl-Counil-Ouest.

8. S. Mauné, Campagne d'évaluation archéologique sur la villa gallo-romaine de L'Auribelle-Basse à Pézenas (Héraull), document final de synthèse, 
Grâce à une série de tranchées réalisées à la pelle mécanique sur toute l'emprise accessible, on a pu confirmer la taille relativement réduite du secteur bâti et organiser les campagnes de fouille ultérieures en sectorisant les vestiges selon leur emplacement et leur fonction. Près de $20 \%$ du terrain disponible ont été décapés et le maillage des tranchées ne semble pas avoir laissé passer de structure archéologique importante. En 2001, c'est toute la partie arrière du site, correspondant à une zone de dépotoirs et de structures annexes à l'habitat, qui a fait l'objet d'un décapage de $1300 \mathrm{~m}^{2}$ environ. La fouille exhaustive du comblement de plusieurs fosses a livré un très abondant mobilier, tandis qu'à l'extrémité nord du secteur décapé on a pu mettre au jour l'extension du secteur bâti et la fondation de l'aqueduc qui alimentait le site ${ }^{9}$. C'est l'une de ces fosses (FS 1043) installées à l'arrière des bâtiments qui, nous l'avons précisé plus haut, a livré l'essentiel des fragments de la plaque en bronze. En 2002, un décapage de $1500 \mathrm{~m}^{2}$ a permis la mise au jour d'une série de constructions, de fosses-dépotoirs et surtout d'un moulin hydraulique installé dans le prolongement de l'aqueduc. Un second aqueduc, alimentant probablement des installations thermales, a également été identifié.

Bien que très arasés en raison de la faiblesse de la couverture pédologique, les vestiges en place autorisent à restituer une partie du plan dont le phasage précis est en cours (fig. 3).

L'ensemble du dossier concernant L'Auribelle-Basse montre donc que nous avons affaire à un site qui présente un intérêt certain puisque, malgré une superficie apparemment peu étendue, il a livré en abondance des fragments de marbre et des éléments appartenant à des aménagements luxueux que l'on n'a pas l'habitude de rencontrer sur des gisements de cette taille. La découverte d'un texte sur bronze semble également être révélatrice de ce particularisme : peu fréquentes dans le territoire de la cité de Béziers, les inscriptions, presque toujours funéraires ou votives, utilisent en effet la pierre comme support quasi exclusif.

Ces observations, que renforcent encore les analyses en cours portant sur les différentes catégories de mobilier exhumées, nous conduisent à nous interroger sur le statut de ce site et sur son évolution architecturale. S'agit-il d'une villa succédant, après plusieurs générations, à une simple ferme, après une lente transformation du bâti ? Sommes-

déc. 2000, 104 p.; voir aussi le Bilan scientifique régional du service archéologique du Languedoc-Roussillon, Montpellier, 2000, p. 142-143.

9. S. Mauné, Fouille archéologique programmée sur la villa gallo-romaine de L'Auribelle-Basse à Pézenas (Hérault). Secteurs Nord et Ouest: première campagne, juillet 2001, 55 p. (programme 20 du ministère de la Culture : « Espace rural, peuplement et productions agricoles à l'époque galloromaine $"$ ).
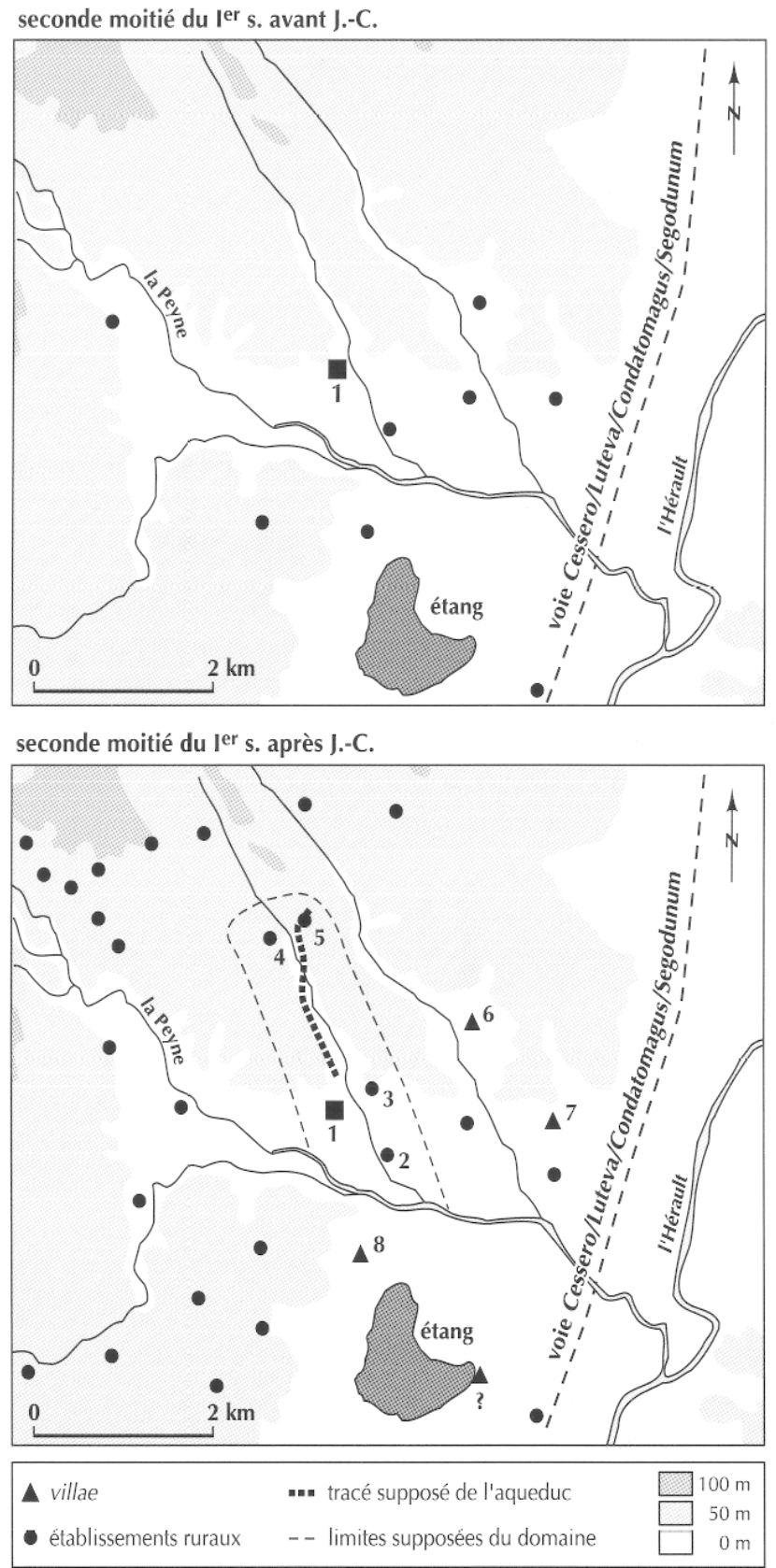

Fig. 2 - L'occupation du sol autour de L'Auribelle-Basse à Pézenas (Hérault) dans la seconde moitié du Irr s. avant J.-C. (2a) et dans

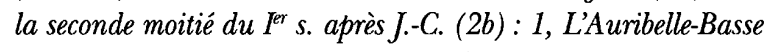
(Pézenas) ; 2, Roquelune (Pézenas) ; 3, Lou Traou-del-Counil-Ouest (Pézenas); 4, Saint-Martin (Caux) ; 5, Camp-Redon (Caux); 6, Saint-Jean-de-Bébian (Pézenas); 7, Chichery (Pézenas); 8, La Perrière (Pézenas) (DAO S. Mauné, CNRS).

nous en présence d'une villa résidentielle précoce, construite dès le dernier tiers du $\mathrm{I}^{\mathrm{er}} \mathrm{s}$. avant J.-C. ? Certaines particularités du plan des constructions, comme l'étroitesse du grand bâtiment occidental, peuvent-elles nous orienter 


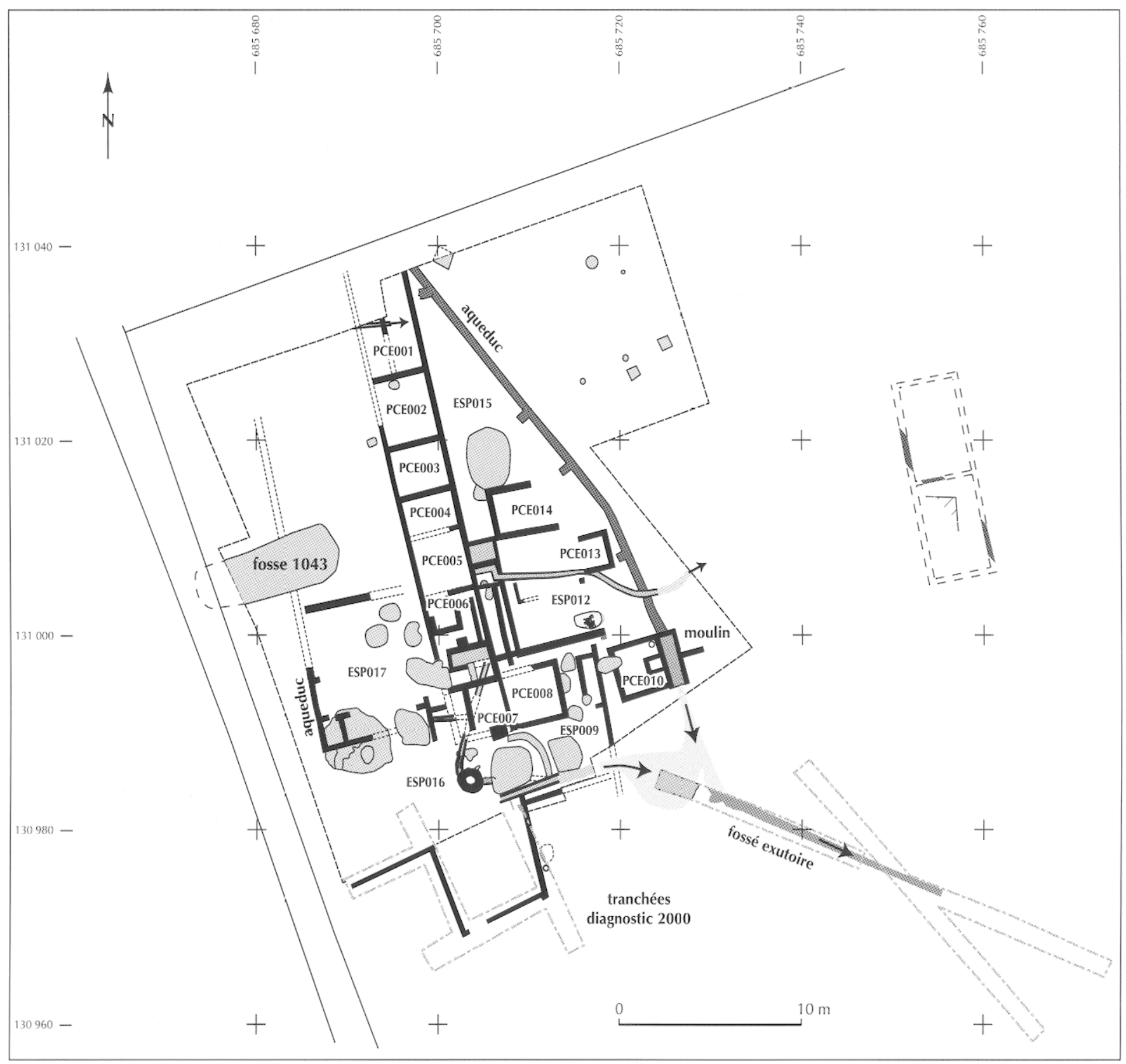

Fig. 3 - Plan provisoire des structures archéologiques mises au jour en 2000-2002 à L'A uribelle-Basse (Pézenas): $P C E$, pièce ; ESP, espace (DAOS. Mauné, CNRS).

vers une autre interprétation, et laquelle ? La chronologie de sa fondation doit-elle être mise en relation avec la déduction coloniale de Béziers, intervenue en 36 avant J.-C. ? Qui sont les occupants de cet établissement ?

L'analyse préliminaire des fragments de la plaque en bronze trouvés sur le site peut apporter quelques orientations et pistes de recherche qui montrent, notamment, l'importance de la société indigène dans ces phénomènes complexes liés à la romanisation de la Gaule transalpine.

\section{LES FRAGMENTS DE LA PLAQUE EN BRONZE ET LE TEXTE}

À elle seule, la découverte de l'existence d'une plaque en bronze est un élément intéressant, car ce type de support est en général réservé à des documents officiels. La péninsule Ibérique en a fourni un large échantillonnage: lois municipales, tables d'hospitium et de patronat, décrets ou décisions de représentants du pouvoir 


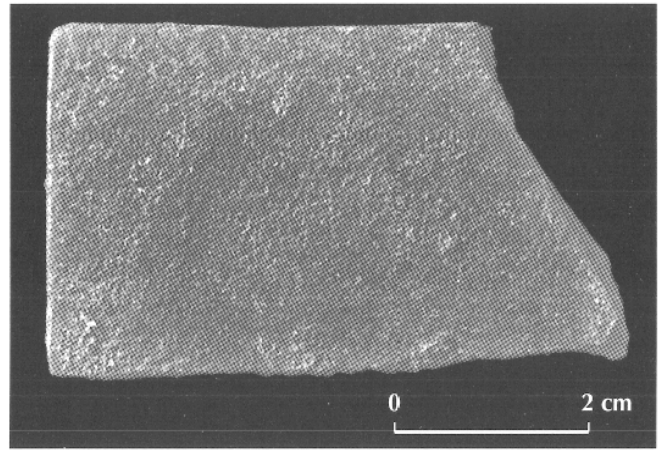

Fig. 4 - Fragment 1 de la plaque en bronze de L'Auribelle-Basse à Pézenas (photo S. Mauné, CNRS).

romain et même diplômes militaires. La Gaule Narbonnaise n'apporte pas autant en quantité ou en diversité : le décret de Digne-Thoard (ILN, Digne, 3), la loi du flaminat provincial (CIL, XII, 6038), une plaque de bronze d'Arles portant le texte d'un hommage public $(A E, 1988,859$ et 1992 , 1182).

Le nombre des fragments s'est accru depuis la première présentation du document, qui s'était accompagnée de la publication d'une brève notice signalétique (Christol, Mauné, 2000). Initialement on ne disposait que de deux fragments. À présent on en possède huit, mais trois sont anépigraphes. Parmi ceux-ci, deux correspondent à des angles, l'angle supérieur gauche et l'angle inférieur droit. Cependant, ce dernier apporte beaucoup lorsque l'on examine la disposition du texte et son contenu.

\section{FraGment 1 (fig. 4)}

On peut considérer qu'il correspond au bord supérieur gauche. C'est un petit fragment ayant la forme d'un trapèze rectangle, de dimensions réduites. Hauteur: $5,7 \mathrm{~cm}$; largeur : $3,3 \mathrm{~cm}$; épaisseur : $0,5 \mathrm{~cm}$. Il est anépigraphe.

\section{Fragment 2 (fig. 5)}

Il appartient à la bordure supérieure, dans une partie médiane. C'est un fragment de forme irrégulière et de dimensions moyennes qui comportait, sous une large bande anépigraphe correspondant à la marge supérieure, une ligne inscrite, dont ne subsistent que huit lettres. Par rapport à l'ensemble de l'inscription, c'est à cette ligne que se trouvent les lettres de la plus grande dimension. Hauteur max. : $6,4 \mathrm{~cm}$; largeur max. : 7,3 cm ; épaisseur : $0,5 \mathrm{~cm}$. Hauteur des lettres : $1,8 \mathrm{~cm}$. On lit :

- - - ]EIILLVES[- - -

On peut se demander s'il ne convient pas de relever l'existence d'un point inséré à l'intérieur de la quatrième lettre (L), séparant ainsi deux mots inscrits, d'où la possi-

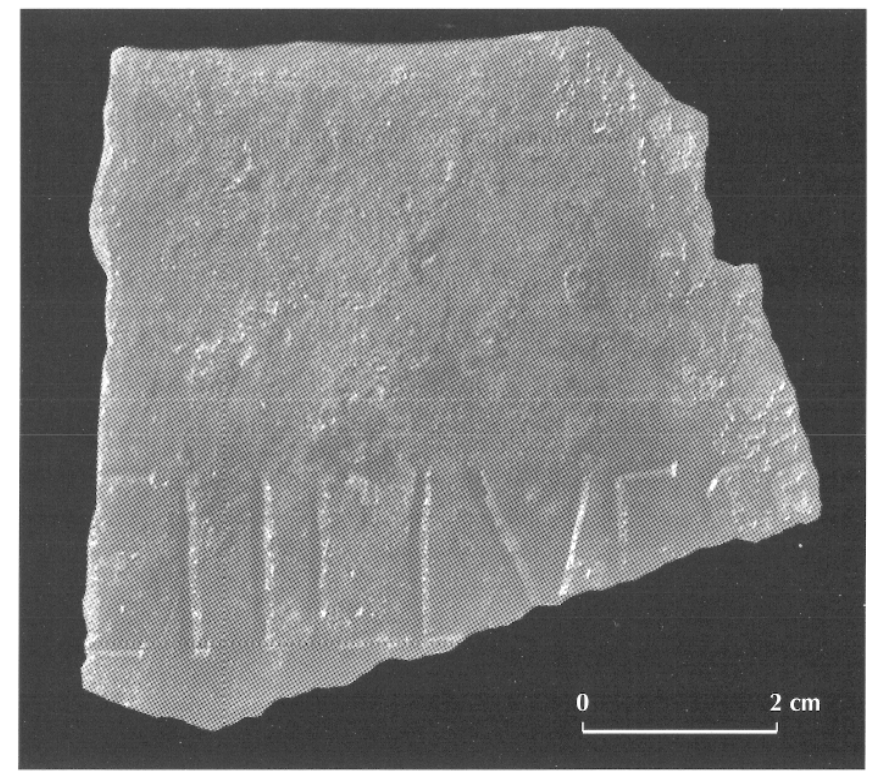

Fig. 5 - Fragment 2 de la plaque en bronze de L'Auribelle-Basse à Pézenas (photo S. Mauné, CNRS).

bilité d'une lecture - - - ]EIIL•LVES[- - . La première lettre est $\mathrm{E}$ : le trait horizontal supérieur est un peu plus allongé que le trait inférieur. Les empattements à la base des deux lettres suivantes (I) sont bien marqués. La base horizontale des $\mathrm{L}$ qui suivent est allongée et se redresse quelque peu. La lettre suivante est $\mathrm{V}$, ouverte de façon dissymétrique vers la gauche. Puis on lit vraisemblablement $\mathrm{E}$ plutôt que $\mathrm{F}$, et enfin $\mathrm{S}$. Alors le texte inscrit pouvait se continuer au-delà de la cassure.

\section{FRAGMENT 3 (fig. 6)}

Fragment de forme très irrégulière. Il correspond à une partie du texte qui se trouvait au cour de la plaque. Hauteur max. : 4,3 cm ; largeur max. : 4,9 cm ; épaisseur : $0,5 \mathrm{~cm}$. Hauteur des lettres à la ligne $1: 1,4$ à $1,5 \mathrm{~cm}$; à la ligne 2 : 1 à $1,2 \mathrm{~cm}$; à la ligne $3: 1 \mathrm{~cm}$. On lit :

$$
\begin{aligned}
& \text { - - - ] PRO[-.- } \\
& \text { - - - }] \text { NITIAC[- - } \\
& \text { - - }] \text { CABIRV[- - }
\end{aligned}
$$

À la ligne 1, un vacat précède les trois lettres conservées, donnant ainsi le début du mot pro[-- - ]. À la ligne 2, après C pourrait peut-être aussi apparaître un point suivi d'un vacat indiquant que le mot s'interrompait, mais la trace est bien moins nette que sur le fragment 2. Pour l'un comme pour l'autre, c'est à partir d'un examen de l'inscription du texte sur le métal que l'on pourrait décider, mais pour l'instant ceci est impossible car les éléments dont on dispose ne sont pas suffisamment importants. À la ligne 3, les rapprochements onomastiques imposent de lire le mot Cabiru[s]. 


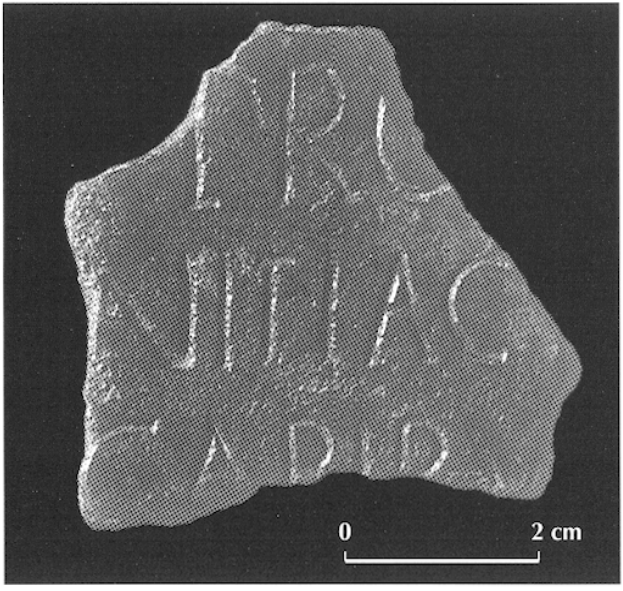

Fig. 6 - Fragment 3 de la plaque en bronze de L'Auribelle-Basse à Pézenas (photo S. Mauné, CNRS).

\section{FraGment 4 (fig. 7)}

Fragment de forme approximativement triangulaire, qui correspondait aussi à une partie du texte se trouvant au cœur de la plaque. Il a déjà été publié (Christol, Mauné, 2000). Hauteur max. : 5,3 cm; largeur max.: 4,9 cm; épaisseur: $0,5 \mathrm{~cm}$. La hauteur des lettres diminue peu à peu; à la ligne $1: 1 \mathrm{~cm}$; à la ligne $2: 1 \mathrm{~cm}$; à la ligne 3 : $0,9 \mathrm{~cm}$; à la ligne 4 , les lettres sont incomplètes. On lit :

- - ]EPI [-..

-. - ] CINTV[--

- - - ]I (ou V)OLCO[- -

-. - ]VI[-.-

À la ligne 1, la première lettre pourrait être un $\mathrm{E}$ dont la base n'a été soulignée que par un étroit tiret. À la ligne 2, peut-être que le $\mathrm{C}$ qui semble commencer un nom de personne était précédé d'un vacat assez ample (comme à la ligne $1 \mathrm{du}$ fragment 3). À la ligne 3 , la première lettre apparaît sous forme d'une haste presque verticale. Il pourrait s'agir d'un I ou même d'un V ouvert de façon très dissymétrique vers la gauche (on le rapprochera du fragment 2). À la ligne 4, la première lettre conservée est aussi un $\mathrm{V}$ ouvert de façon dissymétrique vers la gauche.

\section{FraGment 5 (fig. 8)}

Fragment ayant approximativement la forme d'un rectangle. Il se plaçait sur le bord gauche de la plaque, car la marge anépigraphe de $3,7 \mathrm{~cm}$ est nette. Hauteur: $3 \mathrm{~cm}$; largeur: $5,3 \mathrm{~cm}$; épaisseur : $0,5 \mathrm{~cm}$. Hauteur des lettres à la ligne $1: 1 \mathrm{~cm}$; les lettres de la ligne 2 sont incomplètes. On lit :

$$
\mathrm{D}[---
$$$$
\text { VR (ou B) [- - - }
$$

À la ligne 1, la panse du D se referme un peu au-dessus du départ de la haste initiale. À la ligne 2 , la première lettre

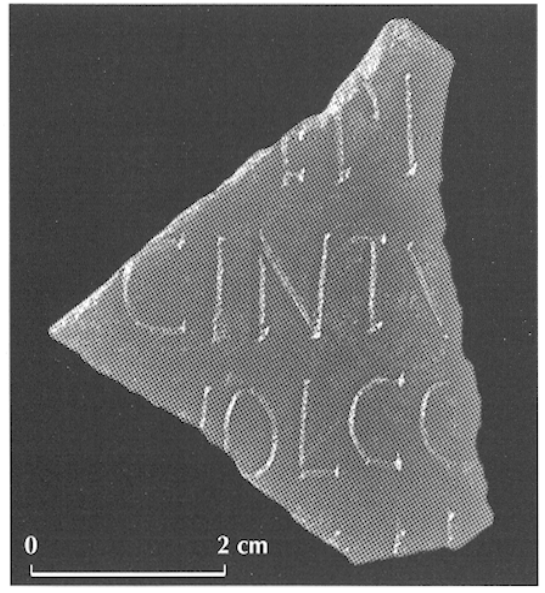

Fig. 7 - Fragment 4 de la plaque en bronze de L'Auribelle-Basse à P'ézenas (photo S. Mauné, CNRS).

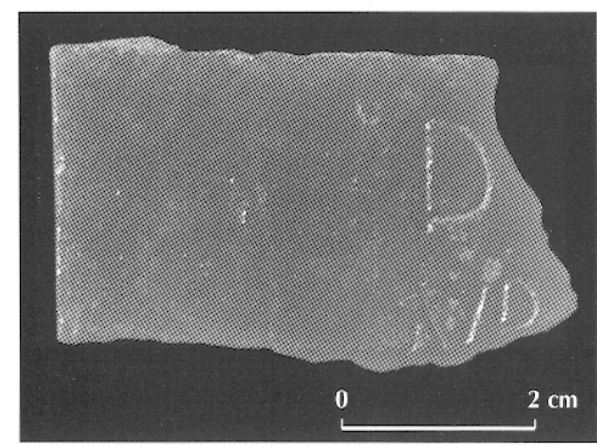

Fig. 8 - Fragment 5 de la plaque en bronze de L'Auribelle-Basse à Pézenas (photo S. Mauné, CNRS).

est un V, mais il n'est pas aussi ouvert de façon dissymétrique qu'ailleurs. Cette lettre est suivie d'un R ou d'un B.

\section{FraGment 6 (fig. 9)}

Fragment ayant approximativement la forme d'un losange allongé vers la droite. La ligne qui était gravée était séparée de la ligne précédente par un espacement un peu supérieur à l'interligne courant. Le mot qui était gravé était aussi isolé sur la ligne par rapport au mot précédent. Ce fragment se positionne par rapport à un fragment anépigraphe, et l'on peut considérer qu'il s'agit de la dernière ligne du texte, ainsi que du dernier mot du texte au bas de la plaque. Hauteur max. : $5,4 \mathrm{~cm}$; largeur max. : $8 \mathrm{~cm}$; épaisseur: $0,5 \mathrm{~cm}$. Hauteur des lettres: $1,1 \mathrm{~cm}$. Le L majuscule est cependant un peu plus grand : $1,2 \mathrm{~cm}$. On lit :

$$
\text { -.- - LEGATEI[- - - }
$$

Le trait transversal du L est nettement dessiné. Il est posé parfaitement à l'horizontale, à la différence de ce que l'on constate sur le fragment 2. Le $\mathrm{E}$ est plus long en bas. Le $\mathrm{T}$ est légèrement dissymétrique à gauche. 


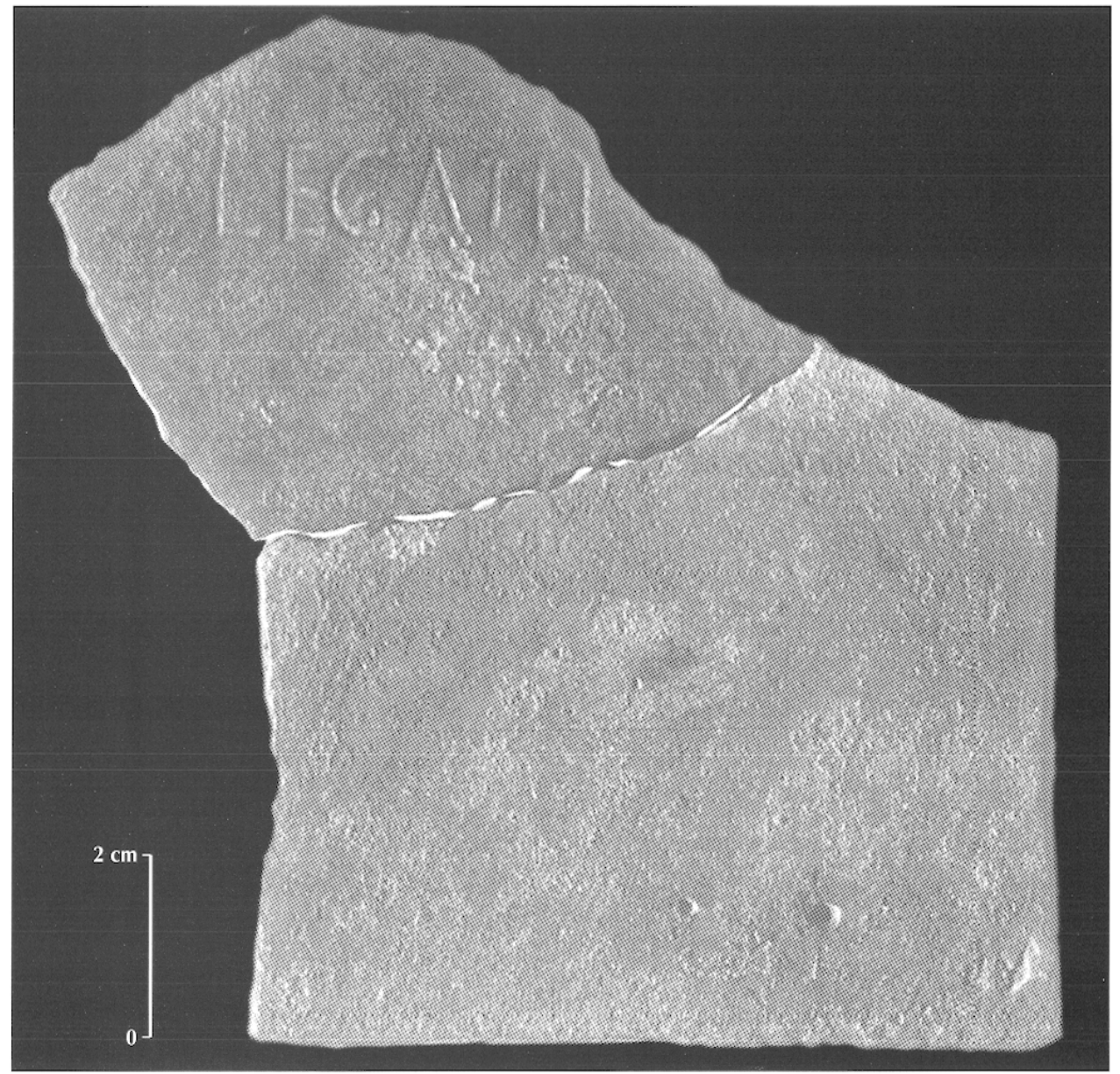

Fig. 9 - Fragments 6 et 7 de la plaque en bronze de L'Auribelle-Basse à Pézenas (photo S. Mauné, CNRS).

\section{FraGment 7 (fig. 9)}

Fragment de forme irrégulière, non inscrit. Il s'adapte au fragment 6 et constitue l'angle inférieur droit de la plaque. Hauteur max. : 7,7 cm; largeur max. : 9,4 cm; épaisseur : $0,5 \mathrm{~cm}$.

\section{Fragment 8}

Le petit fragment anépigraphe (angle supérieur droit ou inférieur gauche) provenant de l'US 1029, mis au jour en juillet 2000 , a été momentanément égaré et ne peut être pris en compte.

Il est difficile d'évaluer les dimensions totales de cette plaque. Le texte a été gravé en ménageant apparemment une marge de $4,1 \mathrm{~cm}$ en haut, de $3,7 \mathrm{~cm}$ à gauche et de $5,3 \mathrm{~cm}$ à droite ${ }^{10}$. En revanche, la marge inférieure était de

10. Cette mesure étant prise sur le fragment 6 , entre la dernière lettre de legatei et le bord extéricur du fragment 7. On admet donc ici, sans pouvoir lc démontrcr, quc la limitc droitc du texte était alignée sur le $i$ de legatei.
$8,6 \mathrm{~cm}$. Si l'on admet que le texte a été gravé en lettres peu à peu décroissantes, le fragment 3 se place au-dessus des fragments 4 et 5 . Comme on le verra un peu plus bas, on pourrait préciser le positionnement des fragments $3,4,5$ et 6 , en les plaçant dans la partie inférieure de la plaque.

Plusieurs noms de personnes apparaissent.

D'abord on évoquera le nom Cabiru[s]. C'est celui dont la lecture est la plus évidente (fragment 3). Cabirus ou Kabiros sont enregistrés comme anthroponymes dans la plupart des recueils ${ }^{11}$. Holder enregistre d'abord Cabiriacus (issu de Cabiros) ${ }^{12}$, puis Caburus ${ }^{13}$, qui doit être un équivalent, avant d'insérer dans son ouvrage Cabirus lui-même ${ }^{14}$. En général dans ces ouvrages on fait aussi allusion au gentilice Cabirius, attesté à Valence pour un citoyen romain

11. Voir Evans, 1967, p. 317 jusqu'à l'ouvrage récent de J. Degrave, 1998, p. 122. Mais Delamarre ne l'enregistre pas (Delamarre, 2001).

12. Holder, I, col. 665.

13. Holder, I, col. 667 ; che\% les Hélviens : César, $B G$, I, 47, 4; VII, 65, 2.

14. Holder, III, col. 1025. 
d'origine locale s'appelant Sex(tus) Cabirius Iullinus ${ }^{15}$, alors que Cabirus apparaît aussi à Cologne pour une personne originaire de la cité des Viromanduens ${ }^{16}$. En Gaule méri-

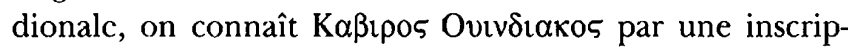
tion gallo-grecque de Cavaillon ${ }^{17}$. Surtout, à proximité de Pézenas, sur le site de l'antique Cessero, on connaît Annia Kabira. Par sa dénomination elle indique la possession du droit de cité romaine. Mais il s'agit d'une personne d'origine provinciale, mariée à Divecillus et mère de Canava ${ }^{18}$. Nous disposons donc avec Cabiru[s] de l'attestation d'un anthroponyme indigène. Il est plus difficile de rattacher à un lieu précis l'attestation $Q$. Cabiri peinte sur une amphore Dressel 20 provenant de Sainte-Colombe, Rhône (Helly et al., 1986, p. 130).

On retiendra aussi le début du nom Cintu[---]. Si notre lecture est bonne, l'élément Cintu-apparaît au début d'un nom composé. Le mot signifie « celui qui est le premier " ${ }^{19}$. C'est un élément entrant fréquemment en composition, qui a donné Cintugenus et Cintugnatus ${ }^{20}$. Dans la Gaule méridionale, on trouve Cintugnatus Boudi f(ilius) chez les Hélviens ${ }^{21}$, et chez les Volques Arécomiques il vient d'apparaître depuis peu ${ }^{22}$; il existe des formes plus courtes, tel le nom Cintullus qui apparaît trois fois dans la même inscription de la cité de Nîmes: Tertius Cintulli f(ilius); Cintullus Ateponis f(ilius); Quinta Cintulli f(ilia ${ }^{23}$. L'élément nominal a aussi fourni le gentilice Cintius/Cintia ${ }^{24}$. Dans l'épigraphie

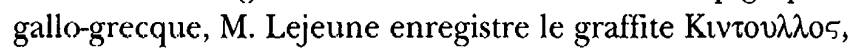
un hypocoristique en - ullus $^{25}$ : on peut donc placer tous ces éléments anthroponymiques dans une totale continuité, de l'épigraphie gallo-grecque à l'épigraphie latine.

Nous retirons de ces premières observations que la plaque pouvait comporter une liste de dénominations

15. ILGN, 258 .

16. CII, XIII, 8342 ; voir, à propos du caractère celtique, Weisgerber, 1968, p. 74, 192, 199, 223 et 224.

17. Lejeune, 1985, p. 150 et L.ambert, 1995, p. 82.

18. $A E, 1969-1970,383$; Massal, Nony, 1977 ; sur les questions de dénomination en relation avec l'application du droit latin, voir Christol, 1989, p. 74-75.

19. Dottin, 1920 , p. 100 ; Vendryes, 1924 , p. $36-39$; Evans, 1967 , p. 179-180; Degrave, 1998, p. 152 et enfin Delamarre, 2001, p. 98.

20. Schmidt, 1957, p. 172.

21. CIL, XII, 2665. L'attestation du mot dans IIGGN, 40 (cité de Riez) doit être écartée d'après la révision de l'inscription : ILN, Riez, 25.

22. Christol dir., 1992, p. 28-34, $\mathrm{n}^{\circ} 6$.

23. CIL, XII, $3944=H G L, \mathrm{XV}, 1203$; Holder, I, col. 1023 et III, col. 1224

24. CIL, XII, $3518=I A N, 310=H G L, \mathrm{XV}, 750:$ Cintia Honorata, à Nîmes ; Holder, I, col. 1021 ; Evans, 1967, p. 177-180.

25. I.cjeune, 1985, p. 414 : G-278. de personnes. On pourrait ainsi envisager que les maigres bribes de mots qui apparaissent sur les fragments 4 et 5 entreraient sans trop de difficulté dans cette catégorie des noms attestés dans la province. Mais leur restitution demeure, - faut-il le préciser ? - encore aléatoire. Toutefois les lettres -- -]EPI[--- pourraient correspondre au nom propre Atepillus/Atepilla. On connaît à Nîmes une femme du nom d'Atepilla Atessatis fil( $(\mathrm{ia})^{26}$, et le nom Atpillus apparaît aussi dans l'anthroponymie celtique ${ }^{27}$. À environ $15 \mathrm{~km}$ au nord de Pézenas, sur l'atelier augustéen de Dourbie/SaintBézard à Aspiran, un certain Atep(o) ou Atep(.) est connu par le biais de son estampille, apposée sur la lèvre d'une partie des amphores Pascual 1 locales ${ }^{28}$.

De même, VOLCO[--- (préféré à IOLCO[--) peut aussi trouver une correspondance dans le glossaire anthroponymique celtique. Outre le nom même des Volques, on connaît par César Catuvolcus, qui dominait avec Ambiorix chez les Eburons ${ }^{29}$.

Ailleurs ce ne sont que des éléments insignifiants. Mais si, sur le fragment 5, les lettres VR[--- (plutôt que VB[---) constituaient le début d'un mot, on pourrait rapprocher celui-ci du nom Vritto/Vrittus qui est attesté dans la cité de Nîmes, avec Boudus Valerius Vrittonis f(ilius) ${ }^{30}$. Il s'agit d'un hypocoristique qui entre dans la série des mots formés sur le thème irit $u^{-}$: il s'en trouve une certaine variété dans la province. Dans l'épigraphie gallo-grecque on connaît le

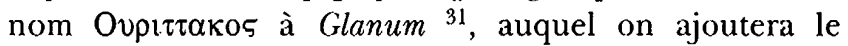

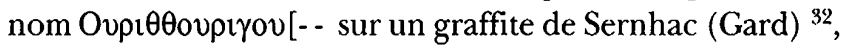
ainsi qu' $[\mathrm{O}] v \rho \imath \theta[0 v] \lambda \lambda \iota[\varsigma]$ à Cabrières-d'Avignon (Vaucluse) ${ }^{33}$. Mais à Nîmes apparaît aussi, comme on l'a dit plus haut, le nom Vritto, utilisé comme patronyme dans une dénomination de citoyen romain. Ailleurs, dans la Narbonnaise, comme dans d'autres provinces gauloises,

26. CIL, XII, 3429 et add. $=H G L, \mathrm{XV}, 646$. Holder, I, col. 257 ; voir aussi Alepilos: Holder, I, col. 257 et III, col. 717 ; Schmidt, 1957, p. 138 ; Evans, 1967, p. 143.

27. Holder, I, col. 267-268 et III, 728; Evans, 1967, p. 143 et 198.

28. Sur cet atelier et cettc marque, voir Genty, Fiches, 1978 et Mauné, 1998 , p. 208-209, 301-302 et 306-307.

29. César, $B G, \mathrm{~V}, 24,4$ et 26,1 ; VI, 31, 5. Holder, I, col. 865 et III, col. 441 ; Schmidt, 1957, p. 300, qui fait observer que l'on se trouve alors dans le contexte d'un peuple germanique; Delamarre, 2001, p. 94. Sur Volio, Degrave, 1998, p. 459 ; Delamarre, ibid., p. 274-275. Sur Catu-, Degrave, ibid., p. 144. A propos du nom des Volques, Lambert, 1995 , p. 34 .

30. CIL, XII, $3478=H G L, \mathrm{XV}, 694$ (à corriger) ; Holder, III, col. 42.

31. Icjeune, 1985 , p. 86-88 : G-68. Il s'agit d'un hypocoristique en -aco: voir aussi Lambert, 1995, p. 83.

32. Lejeune, 1985 , p. 302-304 : G-217, c'est un nom composé.

33. Lejeune, 1985 , p. 137-138: G-111, il s'agit d'un adjectif patrony mique en -io-; voir aussi p. 71-72: G-62 (Saint-Blaise). 
apparaissent plusieurs attestations du gentilice Vrittius, ou des noms de personnes dérivés d'vrit $u^{-}{ }^{34}$.

Quant au mot -.--]nitiac[---, il peut aussi entrer dans cette catégorie des anthroponymes celtiques. Le suffixe -aco caractérise un certain nombre d'anthroponymes comme l'avait déjà constaté $G$. Dottin ${ }^{35}$ : nous avons déjà relevé

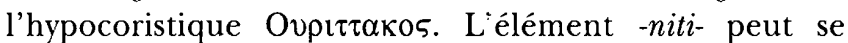
retrouver dans le nom des Nitiobroges ${ }^{36}$, mais le mot pouvait comporter aussi un préfixe. De plus, on peut hésiter entre un nom personnel, tel que nous venons de l'envisager, et un adjectif patronymique en -aco, comme il en existe aussi dans l'anthroponymie celtique ${ }^{37}$ : dans ce cas, les lettres conservées correspondraient au second élément d'une dénomination bimembre.

Dans le corps du texte, tel qu'il semble se développer sur la plaque de bronze, apparaîtraient donc des noms de personnes, formant même une liste importante. Échapperait à cette caractéristique le contenu de la première ligne au moins, c'est-à-dire le début du texte. Mais le contenu de ce fragment 2 demeure énigmatique pour l'instant. De même, sur le fragment 3 , qui semble précéder les fragments 4,5 et 6 , et qu'il convient de positionner un peu plus haut que ces derniers, les lettres ---]pro[-- résistent à tout rapprochement avec l'anthroponymie celtique. S'agirait-il d'un mot latin, nom commun ou nom propre ? En tout cas, à la dernière ligne du texte, l'ultime mot, legatei, nominatif pluriel de legatus sans aucun doute, est incontestablement latin ${ }^{38}$. Il faut envisager que si les caractéristiques de l'anthroponymie renvoient au monde celtique, le texte n'était certainement pas rédigé en langue gauloise.

Le dernier mot du texte oriente l'interprétation dans une voie nouvelle. Dans un premier temps, - mais avec très peu d'éléments il est vrai - nous avions envisagé que le texte pouvait correspondre à un document militaire inscrit dans le bronze, comme il est écrit dans l'inscription relatant les récompenses du cavalier santon qui avait servi dans l'aile Atectorigiana ${ }^{39}$. Se renforcent désormais les données qui rapprocheraient le document d'une table de patronat ou

34. Voir Schmidt, 1957, p. 301 ; Evans, 1967, p. 388 ; Lejeune, 1971, p. 80-83 et 1985 , p. 445 et 456 ; inventaire critique des attestations par Gascou, 2000, p. 223-227 (à propos de Cll, XII, 615). On pourrait aussi le rapprocher d'VRCIVS, attesté sur un graffite d'Ensérune (Clavel, 1970 , p. 570 , d'après Jannoray, 1955, p. 448-449).

35. Dottin, 1920, p. 107-108; voir aussi Lambert, 1995, p. 82-83.

36. Schmidt, 1957, p. 249 ; Lambert, 1995, p. 32.

37. Lejeune, 1985, p. 453 ; voir aussi supra, p. 378 note 17 , le cas de

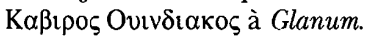

38. Le contexte linguistique est différent dans Lejeune, 1988, p. 13-24: E 1, lorsqu'apparaît un KUITOS LEKATOS.

39. CII, XIII, 1041 ; voir à ce sujet les commentaires de P. Le Roux (1987). Sur cette interprétation, voir Christol, Mauné, 2000, p. 365. d'une table d'hospitalité, car celles-ci comportaient régulièrement la mention de légats, envoyés du peuple ou de la communauté concluant les relations de clientèle avec le puissant personnage qui désormais jouait le rôle de patron ou bien échangeait des services ${ }^{40}$. Très souvent l'inscription précise : egerunt legati, et suit alors une liste plus ou moins longue de noms de personnes représentant la communauté qui se rapprochait ainsi d'un personnage considéré comme important ${ }^{41}$. Mais, dans un cas au moins, le pluriel legati ne précède pas mais clôt l'énumération : il s'agit d'une table d'hospitalité qui révèle l'accord passé entre la cité d'Apisa maius, en Afrique, et le chevalier romain C(aius) Silius C(ai) f(ilius) Aviola, tribun militaire et préfet des ouvriers. Le verbe egerunt ouvre la dernière phrase, il est suivi d'une liste assez fournie de noms indigènes; enfin le mot legati, un peu séparé de la dernière dénomination, vient parachever la phrase et le texte ${ }^{42}$. Un coup d'œil dans l'ouvrage sur les tables de bronze provenant d'Andalousie montre que ce terme peut se trouver en plusieurs emplacements dans la phrase finale : dans un cas, il semble appartenir à la formule initiale, si la restitution eg[erunt legati] s'impose, ce qui semble vraisemblable ${ }^{43}$, mais dans un autre cas il se pourrait que le mot legati, à présent disparu en raison des mutilations de la plaque, se trouvât seul au centre de la dernière ligne ${ }^{44}$. S'il fallait envisager de la sorte la construction de la dernière phrase $\mathrm{du}$ texte inscrit sur la table provenant des environs de Pézenas, il faudrait en conclure que les fragments 3, 4, 5 et 6 appartiennent à la partie inférieure de la plaque, constituant l'énumération des représentants du peuple ou de la cité qui conclut le contrat d'hospitium. En revanche, nous manqueraient pour l'instant des éléments de la partie supé-

40. En principe clientela et hospitium sont des notions distinctes. Mais le développement de la puissance romaine conduisit aux mêmes effets pratiques (Badian, 1958, p. 11-13 et p. 154-155).

41. ILS, 6102, 6104, 6106, 6107, 6108. Variation dans ILS, 6103 : agentibus legatis. Autre variation dans la tessère de Montealegre (Hispania citerior), per legatos: $A E, 1985,581=A E, 1988,764$; texte aussi dans Castillo, 1989, p. 283-284.

42. CIL, V, 4291 (ILS, 6099) = I. Ital., X, V, 1146. Ce document a suscité récemment une ample bibliographie : Gregori, 1990 et 1991 ; Condina, Foraboschi, 2000.

43. CIL, II, 1242 ; González Fernández, 1990, p. 189-190, n 18 avec pl. XXVIII. Même formulation dans le texte des p. 195-197, $\mathrm{n}^{\circ} 21$. Autres exemples dans la péninsule Ibérique: d'abord la Tabula de Cañete $(A E, 1983,530$, voir Castillo, 1989, p. 281-283), puis la Tabula Lougeiorum $(A E, 1989,431=A E, 1984,553$, voir Castillo, 1989, p. $279-280)$.

44. $A E, 1955,21$; González. Fernández, 1990, p. 191-192, n 19 avec pl. XXIX. On relèvera que dans $A E, 1962,287$, repris par ce dernier, p. 193-194, le mot leg(ati) sépare sur une ligne propre les deux dénominations des envoyés de la cité de Munigua, placées respectivement audessus et au-dessous. 
rieure du texte, sauf pour la première ligne, cependant encore énigmatique.

$$
*
$$

Les données qui semblent ressortir de ce texte sont encore extrêmement fragmentaires. Celui-ci est en effet trop incomplet pour que l'on envisage avec sûreté l'ensemble de son contenu, notamment l'identification du personnage principal et de la communauté indigène qui concluait avec lui le contrat d'hospitium: ce sont des éléments importants qui conditionnent la compréhension de l'existence en ce lieu de cette plaque de bronze. Sans aucun doute, tout nouveau fragment peut apporter beaucoup: tant sur l'anthroponymie de la Gaule méridionale que sur les pratiques politiques et sociales d'une communauté dont la dénomination seule offrirait une donnée de premier plan, ou bien sur la personnalité qui est impliquée dans la conclusion de ce contrat. Nous en saurions un peu plus sur l'histoire sociale et politique du monde indigène dans la seconde moitié du I ${ }^{\text {er }}$ s. avant J.-C. ou au tout début du ${ }^{\text {er }}$ s. après J.-C. C'est en effet dans ce secteur, correspondant au bassin de la Peyne, affluent de l'Hérault, que l'on place, pour ce qui concerne la période protohistoique, le peuple des Piscinae, et c'est aussi, quelles que soient les vicissitudes de la géographie administrative imposées par le pouvoir romain, un point de référence pour la localisation de l'oppidum latinum de même nom, connu par Pline ( $H N$, III, 37$)^{45}$.

Plus ponctuellement, les conditions de la découverte des huit fragments méritent une attention particulière : deux proviennent des abords immédiats de la partie méridionale du site, les six autres ont été trouvés dans le comblement d'une grande fosse-dépotoir ${ }^{46}$.

Cette répartition ne laisse pas d'étonner. En effet, n'est-il pas surprenant que la plaque ait été brisée en petits morceaux et que ceux-ci aient été soit jetés en marge des bâtiments, soit mêlés aux poubelles se trouvant à l'arrière des constructions ? La chronologie de comblement de cette vaste fosse a pu être fixée, grâce à la présence d'un abondant.

45. Sur cette problématique, voir Christol, 1998 et Mauné, sous presse.

46. Rappelons que parmi ces derniers, l'un a été mis au jour en juillet 2000 lors de l'opération d'évaluation, dans la coupe réalisée dans cette structure en creux ; les cinq autres lors de la fouille du dépotoir, en juillet 2001. mobilier, entre les années 220 et 250. Dans l'état actuel des recherches et conformément à l'image livrée par le mobilier collecté en surface - qui indique un abandon dans le courant du III ${ }^{\mathrm{e}}$ s. -, il s'agirait de l'ensemble le plus récent mis au jour sur le site. De fait, on se trouve ici en présence d'un dépotoir associant déchets culinaires (céramique, faune, objets, etc.) mais également rejets cendreux très denses provenant d'un praefurnium, probablement celui des installations thermales dont on suppose, avec quelques arguments, la présence dans l'aile sud. Cette vaste fosse servait à entreposer les ordures et devait être curée régulièrement, son contenu pouvant être répandu dans les parcelles cultivées ou jeté dans la rivière toute proche. On peut donc considérer qu'elle a servi de réceptacle aux derniers témoignages matériels de l'occupation du site et que l'abandon de celui-ci était programmé. Dans le cas contraire, pourquoi y trouverait-on des fragments de la plaque?

On sait que le bronze avait une certaine valeur pécuniaire et la fonte de la plaque aurait semblé plus logique. Au lieu de cela, elle a été minutieusement brisée et dispersée au moins en deux endroits différents, éloignés de plusieurs dizaines de mètres. La datation de ce « geste » a également son importance puisque nous avons vu que celui-ci est intervenu juste avant l'abandon définitif. Rappelons ici que dans la première publication la chronologie de la plaque avait été fixée, à partir de l'écriture, à la période tardo-républicaine et augustéenne. Les nouveaux éléments acquis, notamment la graphie archaïque legatei, confirment ce point de vue. Cela concorde aussi avec la présence, apparemment exclusive, d'une anthroponymie celtique dans la liste des personnages qui se trouvait à la fin du texte. Il faut donc bien admettre que cette plaque est restée en place durant près de 300 ans.

L'interprétation de ce document exceptionnel peut être vraisemblablement liée à la fondation et à la fonction de l'établissement dont les artefacts les plus anciens renvoient à la seconde moitié du ${ }^{\text {er }}$ s. avant J.-C. De même, il paraît assuré que cette destruction organisée - qui ne permettait en aucun cas de rassembler les fragments et de lire, à nouveau, l'inscription - avait un but bien précis et revêtait un aspect symbolique évident : en brisant cette plaque, on détruisait un texte dont le contenu fixait un certain nombre de droits, peut-être associés à la propriété éminente des terres environnantes mais aussi au statut des occupants de l'établissement de L'Auribelle-Basse. 


\section{BIBLIOGRAPHIE}

\section{Abréviations}

AE: L'Année épigraphique. Revue des publications épigraphiques relatives à l'Antiquité romaine. Paris, Ernest Leroux et PUF, 1899-

BG : César, Bellum Gallicum. Paris, I.es Belles Lettres (coll. des Universités de France).

CIL : Coppus inscriptionum Latinarum consilio et auctoritate Academiae litterarum Regiae Borussicae editum. Berlin, 1898 .

HGL: Histoire générale de Languedoc, par Dom C. Divic: et Dom J. VAisserTe, t. XV. Toulouse, Privat, $1892,1254 \mathrm{p}$.

$H N$ : Pline l'Ancien, Histoire naturelle. Paris, Les Belles Lettres (coll. des Universités de France).

IAN : Inscriptions antiques de Nîmes, par kugène GFrmFr-Dirand, François Germer-Durand, Auguste Aitimfr. Toulouse, Privat, 1893, $1100 \mathrm{p}$.

ILGN: Inscriptions latines de Gaule (Narbonnaise), par Émile EsPÉrandieu. Paris, Ernest I.croux, 1929, 224 p.

ILN: Inscriptions latines de Narbonnaise. Paris, éd. du CNRS, 1985-.

ILS: Inscriptiones Latinae selectae, edidit Hermannus Dessal, I-III. Berlin, Weidmann, 1892-1916.

BADIAN E.

1958 : Foreign clientelae. Oxford, Clarendon Press.

BRUN J.P. avec la collab. de BorRÉANI M.

1999 : Le Var. Paris, éd. Académie des inscriptions et belles-lettres (coll. Carte archéologique de la Gaule, 83/1 el 83/2), 2 vol., 490 p. ct 494 p.

Castullo C.

1989 : « Epigrafia jurídica romana de Hispania en el ültimo decenio: época imperial ", in Epigrafia juridica romana, Actas del coloquio internacional AIEGL, Pamplona, 9-11 de abril de 1987, p. 277-298.

Christol M.

1989 : "Le droit latin en Narbonnaise : l'apport de l'épigraphic (en particulier celle de la cité de Nîmes) ", in Épigrafia jurídica romana, Actas del coloquio internacional AIEGI., Pamplona, 9-11 de abril de 1987 , p. $65-76$.

1998 : "Cités et territoires autour de Béziers à l'époque romaine ", in ClaAvelLF́Vk̂QUF. M., VIGNOT A. (dir.), Cité et territoire, Actes du $2^{c}$ colloquc curopéen de
Béziers, 24-26 oct. 1997. Paris, Presses universitaires franc-comtoises, p. 209-222.

Christol M. (DIR.)

1992 : Inscriptions antiques de la cilé de Nimes (IACN, 1-21). Cahier des musées et monuments de Nîmes, 11,112 p.

\section{Christol M., Mauné S.}

2000 : «Fragments d'une table de bronzc provenant des environs de Pézenas (département de l'Hérault) ». Cahiers $d u$ Centre Gustave-Glotz, 11, p. 364-365.

\section{Clanel. M.}

1970 : Béziers et son territoire dans l'Antiquité. Paris, Les Belles-Lettres (coll. Annales littéraires de l'université de Besançon, 12i.

\section{CLAVEL-LÉvÊQUE M.}

1998 : «Le réseau colonial Bériers C in M. Clavel-Lévêque ", in VIGNOT A. (dir.), Allas historique des cadastres d'Europe. Bruxelles, Commission européenne.

Condina F., Foraboschi D.

2000 : "Africa-Brescia: andate e ritorno? Ancora su Silio Aviola ", in L'Africa romana, Atti del XIII convegno di studio, Djerba, 10-13 dic. 1998. Pubblicazioni del Centro di studi sulle Province Romane dell'Università di Sassari, 16, Sassari, II, p. $1309-1319$

DEGRAVE J.

1998 : Lexique gaulois. Recueil des mots attestés, transmis ou restitués et de leurs interprétations. Mémoires de la Société belge d'études celtiques, $9,522 \mathrm{p}$.

\section{DELAMARRE X}

2001 : Dictionnaire de la langue gauloise. Paris, éd. Errance, 352 p.

DotTin G.

1920 : La langue gauloise. Paris, éd. Klincksieck, $364 \mathrm{p}$.

Evans D. E.

1967 : Gaulish Personal Names. A Study of some continental Celtic Formations. Oxford, Clarendon Press, 492 p.

FICHES J.-L. (DIR.)

1996 : Le III s. en Gaule Narbonnaise: données régionales sur la crise de l'Empire, Actes de la table ronde du GDR 954 du CNRS, Aixen-Provence, I a Baume, 15-16 sept. 1995. Sophia Antipolis, APDCA, 406 p.

Gascou J.

2000 : “Le gentilice VRITTIVS. Remarques sur l'onomastique du pagus Lucretius (territoire oriental d'Arles) ». Zeitschrift für Papyrologie und Epigraphik, 130, p. 223-231.
GenTY P.-Y., FICHES J.-L.

1978 : « L'atelier de potiers gallo-romains d'Aspiran (Hérault), synthèse des travaux de 1971 à 1978 ». Figlina, 3, p. 71-92.

Ginouvez $\mathbf{O}$.

1995 : “Un vaste site rural d'époquc romaine récemment fouillé sur le territoire de la cité de Béziers ", in Clavel-LÉvîque M. Plana-Maliatart R. (dir.), Cité et territoire, Actes du colloque européen de Béziers, 14-16 oct. 1994. Paris, Les Belles-Lettres (coll. Annales littéraires de l'université de Besançon, 565), p. 169-173.

González Fernández J.

1990 : Bronces jurídicos romanos de Andalucía. Consejería de cultura, s.l., 244 p. et XXXIII pl.

GREGorI G. L.

1990 : " Forme onomastiche indigene e puniche ad Apisa Maius, Siagu, Themetra e Thimilaga ", in L'Africa romana, Atti del VII convegno di studio, Sassari, 15-17 dic. 1989. Pubblicazioni del dipartimento di Storia dell'università degli studi di Sassari, 16, Sassari, I, p. 167-196.

1991 : "Gaio Silio Aviola patrono di Apisa Maius, Siagu, Themetra e Thimilaga ", in L'Africa romana, Atti dell'VIII convegno di studio, Cagliari, 14-16 dic. 1990. Pubblicazioni del dipartimento di Storia dell'università degli studi di Sassari, 18, Sassari, I, p. 229-237.

Helly B., Le Bot-Helly A., Liou B.

1986 : «Un dépôt d'amphores Dr. 20 à inscriptions peintes découvert à SainteColombe (Rhône) ". Archaeonautica, 6, p. 121-145.

HoLder A.

1896-1904-1907 : Alt-Celtischer Sprachschatz, I, Il et III [rëimpression Graz, 1961-1962].

JANNORAY J.

1955 : Ensérune: contribution à l'élude des civilisations préromaines de la Gaule méridionale. Paris, De Boccard.

LAMBERT P.-Y.

1995 : La langue gauloise. Paris, éd. Errance, $240 \mathrm{p}$.

LEJEUNe $\mathbf{M}$.

1971 : « Inscriptions lapidaires de Narbonnaise ». Etudes celtiques, 12, p. 21-91.

1985 : Recueil des inscriptions gauloises -I-Textes gallo-grecs. Paris, éd. du CNRS (Suppl. à Gallia, XLV), $460 \mathrm{p}$.

1988 : Recueil des inscriptions gauloises -II, 1 Textes gallo-étrusques, textes gallo-latins sur 
pierre. Paris, éd. du CNRS (Suppl. à Gallia, XLV), $196 \mathrm{p}$.

\section{LE RouX P.}

1987 : " Th. Mommsen et C. Iulius Macer (CIL, XIII, 1041) ", in Mélanges offerls à Monsieur Michel Labrousse. Toulouse, hors série, Pallas, Revue d'études antiques, p. 119-135.

Massal E., Nony D.

1977 : « Inscription romaine à Saint-Thibéry ». Études sur l'Hérault, 8, p. 3-6.

MAUNÉ S.

1998 : Les campagnes de la cité de Béziers dans l'Antiquité (partie nord-orientale), II $s$. $a v .-V T^{e}$ s. apr. J.-C. Montagnac, Monique Mergoil, $532 \mathrm{p}$.

Sous presse : "À propos de Piscinae, Pézenas et des Piscenois : quelques réflexions sur la localisation de l'agglomération antique et sur les oppida latina de la vallée de l'Hérault ", in Hommage à G. Barruol. Lattes, Monographie d'archéologie méditerranéenne.

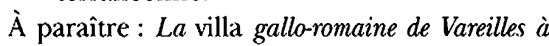
Paulhan (Hérault). Transformations et évolution d'un centre domanial de Gaule Narbonnaise entre la fin du IT s. av. el le début du III s. apr. J.-C. (fouilles 1999-2002). Montagnac, Monique Mergoil (coll. Archéologie et histoire romaine).

\section{Pellecuer C.}

2000 : La villa des Prés-Bas (Loupian, Hérault) dans son environnement. Contribution à l'étude des villae et de l'économie domaniale en Narbonnaise. Thèse de $3^{c}$ cycle, univ. d'Aixen-Provence, 3 vol.
Pellecuer C. (DIr.)

1996 : Formes de l'habitat rural en Gaule Narbonnaise. Sophia Antipolis, APDCA, t. 3.

SCHMIDT K.H.

1957 : «Die Komposition in gallischen Personennamen ». Zeitschrift für Cellische Philologie, 26, p. 33-159 et p. 161-301.

\section{VENDRYES J.}

1924 : «Remarques sur les graffites de la Graufesenque ". Bulletin de la Société de linguistique de Paris, 25, p. 34-43.

WEISGERBER J.-L.

1968 : Die Namen der Ubier. Köln und Opladen, Westdeutscher Verlag (Wissenschaftliche Abghandlungen der Arbeitsgemeinschaft für Forschung in Landes NordrheinWcstfalcn, 34), $479 \mathrm{p}$. 